\title{
Total electron-ion recombination of Fe III
}

\author{
Sultana N. Nahar \\ Department of Astronomy, The Ohio State University, Columbus, Ohio 43210
}

(Received 10 November 1995)

\begin{abstract}
It is shown that a method for calculating unified total electron-ion recombination rates, incorporating both the radiative and dielectronic recombination processes, can be applied to substantially complex atomic systems where a large number of recombination channels need to be coupled in an $a b$ initio manner. Calculations are carried out for the astrophysically important ion Fe III, and total and state-specific recombination rate coefficients are obtained for $e+\mathrm{Fe}$ IV $\rightarrow \mathrm{Fe}$ III, in the close coupling approximation employing the $R$-matrix method. The calculations correspond to a 49-state eigenfunction expansion for the Fe IV core that includes states from the ground configuration $3 d^{5}$ and the excited configurations $3 d^{4} 4 s$ and $3 d^{4} 4 p$ for proper treatment of the electron correlation effects. This is an accurate and detailed atomic calculation for the recombination rates for Fe III. The present rates are up to an order of magnitude lower than the previous values obtained through the Burgess general formula at electron temperatures where dielectronic recombination dominates.
\end{abstract}

PACS number(s): 34.80.Kw, 32.80.Dz, 32.80.Fb

\section{INTRODUCTION}

A unified treatment [1-3] for the total electron-ion recombination in the close coupling approximation is employed for the recombination rate coefficients $\alpha_{R}$ of $e+\mathrm{Fe}$ IV $\rightarrow \mathrm{Fe}$ III. The treatment incoporates both the radiative and the dielectronic recombinations in a consistent and unified manner. It provides the recombination rate coefficients for a wide range of temperatures for all practical purposes, compared to earlier treatments valid in limited temperature ranges and which generally treat radiative and dielectronic recombination (RR and DR) separately in different approximations. The present work reports detailed and accurate calculations for the electron-ion recombination of Fe III, as well as an application of the unified treatment for large complex atomic systems with many channels and relatively strong electron correlation effects.

The separate RR and DR rate coefficients currently in use were both calculated by Woods et al. [4], who employed the photoionization cross sections in the central-field and hydrogenic approximations to obtain the RR rates. For the DR rates they employed the Burgess general formula [5] using oscillator strengths [6] obtained from an atomic structure calculation by Abbott (referenced in Woods et al.). The accuracy of the Fe III oscillator strengths used in the DR calculations is uncertain [J.M. Shull (private communication)]. In a later work, Hahn [7] recalculated the DR rates employing an improved empirical formula based on the Burgess general formula. Arnaud and Raymond [8] combined both these DR rates in their work, which reproduces rates very close to those by Woods et al. [4].

\section{THEORY}

The details of the unified treatment are given in Refs. $[2,3]$. Here a brief outline of the method is presented. The recombination of an incoming electron to the target ion may occur through radiative recombination,

$$
e+X^{2+} \rightarrow X^{+}+h \nu
$$

which is the inverse process of direct photoionization, or through the two-step dielectronic recombination:

$$
e+X^{2+} \rightarrow\left(X^{+}\right) * * \rightarrow\left\{\begin{array}{c}
e+X^{2+} \\
X^{+}+h \nu
\end{array}\right.
$$

where the incident electron attaches itself to the target ion in a quasibound doubly excited state, which leads either to (i) autoionization, a radiationless transition to a lower state of the ion (usually the initial one) and the free electron, or to (ii) recombination with radiative stabilization via decay of the ion core and the bound electron. Coupled channel calculations for photoionization cross sections include both processes, and hence show resonance structures due to the doubly excited autoionizing states. In the close coupling calculations it is important to include all states of the core ion that could contribute significantly to these resonances.

The electron-ion recombination rate coefficient $\alpha_{R}(T)$ can be obtained in terms of the photoionizaton cross sections as

$$
\alpha_{R}(T)=\int_{0}^{\infty} v f(v, T) \sigma_{\mathrm{RC}} d v,
$$

where $\sigma_{\mathrm{RC}}$ is the recombination cross section and $f(v, T)$ is the velocity distribution function, usually chosen to be a Maxwellian. $\sigma_{\mathrm{RC}}$ is related to the photoionization cross section $\sigma_{\mathrm{PI}}$, through detailed balance (Milne relation):

$$
\sigma_{\mathrm{RC}}=\sigma_{\mathrm{PI}} \frac{g_{i}}{g_{j}} \frac{h^{2} \omega^{2}}{4 \pi^{2} m^{2} c^{2} v^{2}} .
$$

Hence $\sigma_{\mathrm{PI}}$, obtained including the autoionizing resonances, essentially provide total $\alpha_{R}(T)$ incorporating both the RR and the DR in a unified manner. However, in the unified treatment the detailed $\sigma_{\mathrm{PI}}$ with resonances must be obtained in the close coupling approximation for a large number of contributing bound states $i_{b}$ of the recombined $e+$ ion system. From the individual contributions the total $\alpha_{R}(T)$ is obtained as 


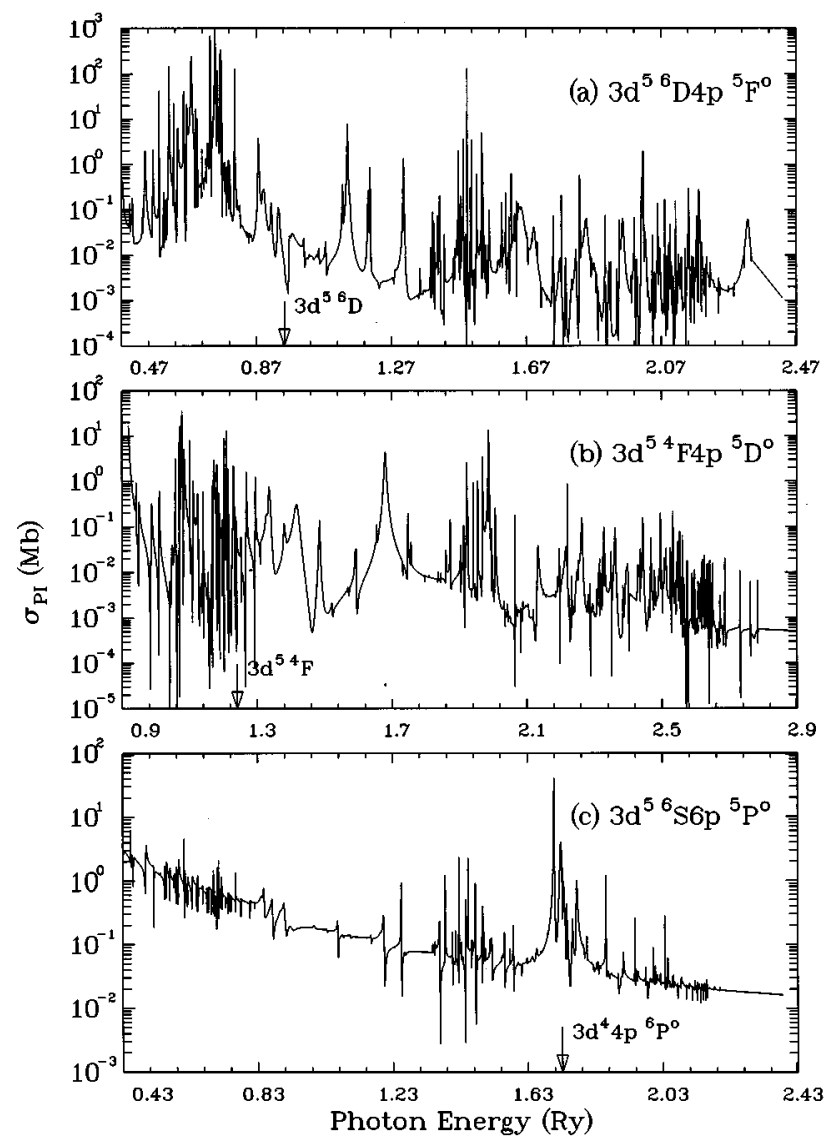

FIG. 1. Partial photoionization cross sections $\sigma_{\mathrm{PI}}$, of (a) $3 d^{56} \mathrm{D} 4 p\left({ }^{5} F^{o}\right)$, (b) $3 d^{54} F 4 p\left({ }^{5} D^{o}\right)$, and (c) $3 d^{56} S 6 p\left({ }^{5} P^{o}\right)$ states of Fe III: Fe III $+h \nu \rightarrow$ Fe IV $+e$. The arrows in (a) and (b) show energy positions for direct photoionization of the states leaving the cores at $3 d^{5}{ }^{6} D$ and $3 d^{54} F$ states, respectively. Panel (c) illustrates the PEC feature with the arrow pointing out the energy position of the optically dipole allowed $3 d^{4} 4 p^{6} P^{o}$ state by the target ground $3 d^{5}{ }^{6} S$ state.

$$
\alpha_{R}(T)=\sum_{i_{b}} \frac{g_{i}}{g_{j}} \frac{2}{k T \sqrt{2 \pi m^{3} k c^{2} T}} \int_{0}^{\infty} E^{2} \sigma_{\mathrm{PI}}\left(i_{b} ; \epsilon\right) e^{-\frac{\epsilon}{k T}} d \epsilon,
$$

where $E=\hbar \omega=\epsilon+I_{p}, \epsilon$ is the photoelectron energy, and $I_{p}$ is the ionization potential. The integration over $\infty$ energy range of the photoelectron is carried out as described in Ref. $[2]$.

In principle, electron-ion recombination takes place to an infinity of states (Fig. 1 of Ref. [2]). To each excited threshold $S_{t} L_{t} \pi_{t}$ of the $N$-electron target ion there corresponds an infinite series of $(N+1)$-electron states, $S_{t} L_{t} \pi_{t} \nu \ell$, where $\nu$ is the effective quantum number, to which recombination can occur. In the unified treatment of electron-ion recombination all these recombining states are considered. The bound states of the recombined $e+$ ion system are divided into two groups, (A) low- $n$ states, where $n$ goes from the ground level to $n_{\max }$ and (B) the high- $n$ states where $n_{\max } \leqslant n \leqslant \infty$. The detailed photoionization cross sections including the autoionizing resonances of all low- $n$ bound states of group (A) are obtained in the close coupling (CC) approximation. For group (B) states DR dominates the recom- bination process and the contributions are primarily in the high temperature range since the incident electron must be sufficiently energetic so as to collisionally impact and excite the target ion to a higher state connected via a dipole transition to the ground state. The contribution from group (B) states is obtained by calculating the collision strengths of DR, $\Omega(D R)$, employing the precise theory of radiation damping by Bell and Seaton [9,2]. The $\Omega(\mathrm{DR})$ are obtained, in a self-consistent manner, in the $\mathrm{CC}$ approximation using the same wave function expansion that is used for the calculation of $\sigma_{\mathrm{PI}}$.

In the $\mathrm{CC}$ approximation, the target or the core ion is represented by an $N$-electron system, and the total wave function expansion $\Psi(E)$ of the $(N+1)$-electron system for any symmetry $S L \pi$ is represented in terms of the target wave functions as

$$
\Psi(E)=A \sum_{i} \chi_{i} \theta_{i}+\sum_{j} c_{j} \Phi_{j}
$$

where $\chi_{i}$ is the target wave function in a specific state $S_{i} L_{i} \pi_{i}$ and $\theta_{i}$ is the wave function for the $(N+1)$ th electron in a channel labeled as $S_{i} L_{i} \pi_{i} k_{i}^{2} \ell_{i}(S L \pi), k_{i}^{2}$ being its incident kinetic energy. $\Phi_{j}$ 's are the correlation functions of the $(N+1)$-electron system that account for short range correlation and the orthogonality between the continuum and the bound orbitals. For the present calculations of $\sigma_{\mathrm{PI}}$ of the group (A) bound states, and $\Omega(\mathrm{DR})$ for the group (B) states, a 49-state wave function expansion is employed [10].

In the following subsections some details of the calculations are presented.

\section{A. Low-n states: Photoionization cross sections}

The recombination rate coefficient of an electron combining with a target ion in the ground state requires the partial photoionization cross sections $\sigma_{\mathrm{PI}}$ of all the recombined states that leave the ion in the ground state. There are 239 such bound states of Fe III in the group (A) low- $n$ bound states (with $n_{\max } \simeq 10$ and $\ell \leqslant 9$ ) that couple to the ${ }^{6} S$ ground state of Fe IV. The partial photoionization cross sections of these states are obtained from large-scale computations for Fe III radiative data [10], in the CC approximation employing the $R$-matrix method as developed for the opacity project (OP) [11] and extended for the iron project [12]. The calculated states were identified through detailed examination of quantum defects by computer code ELEVID. The computation for the recombination rate coefficients for each state was calculated as explained in Ref. [2], employing the computer code RECOMB.

Some results of the cross sections are presented below, pointing out features that are important in the final results for $\alpha_{R}(T) . \sigma_{\text {PI }}$ of the ground state of Fe III, $3 d^{65} D$, is a dominant contributor to $\alpha_{R}$. However, there are excited states that make considerable contributions because of their enhanced cross sections due to autoionizing resonances. Figure 1 presents examples of such states where partial photoionization cross sections of excited $3 d^{56} D 4 p\left({ }^{5} F^{o}\right), 3 d^{5}{ }^{4} F 4 p\left({ }^{5} D^{o}\right)$, and $3 d^{5}{ }^{6} S 6 p\left({ }^{5} P^{o}\right)$ states of Fe III are shown. The cross sections of both states ${ }^{5} F^{o}$ and ${ }^{5} D^{o}$ in Figs. 1(a) and 1(b) show extensive resonances. The arrows in these figures point 
to the threshold energies for direct photoionization of these states leaving the ion cores in states $3 d^{5}{ }^{6} D$ and $3 d^{5}{ }^{4} F$ of Fe IV, respectvely. Owing to the strong coupling effects the cross sections below these target states show dense features belonging to Rydberg series of autoionizing resonances converging on to the target threshold. It is clear that the resonances (with $n \geqslant 10$ ) get narrower, with diminishing background cross section, and the contribution to $\alpha_{R}$ gets to be predominantly of DR type.

At high energies the singular feature in the cross sections that affects the recombination rate coefficients is due to the photoexcitation of core (PEC) resonances [3]. These occur at energies that excite the target ground state to an excited state via a dipole allowed transition, while the outer electron remains essentially a "spectator." The process manifests itself through wide resonances that enhance the background cross sections by orders of magnitude. PEC resonances are pronounced in the photoionization cross sections of excited states along a Rydberg series. This is quite contrary to the behavior expected from the usual hydrogenic scaling of photoionization cross sections for excited states. Figure 1(c) presents cross sections of the $3 d^{5}{ }^{6} S 6 p\left({ }^{5} P^{o}\right)$ state of Fe III for which the background cross sections show smooth variation until the photon energy 1.73 Ryd (marked by the arrow) when the Fe IV state $3 d^{4} 4 p^{6} P^{o}$, is accessible via a dipole transition from the ground state $3 d^{5}{ }^{6} S$; the associated PEC resonance there can be seen clearly. The photoionization of the state $3 d^{5}{ }^{6} S 6 p\left({ }^{5} P^{o}\right)$ better illustrates the PEC feature, though fewer states of the same symmetry contribute to $\alpha_{R}$.

Figure 2 presents the total photoionization cross sections of the ground state $3 d^{65} \mathrm{D}$ of Fe III, also showing photoionization to all included states of the core ion Fe IV [10]. The partial $\sigma_{\mathrm{PI}}$ for the ${ }^{5} D$ ground state leaving $\mathrm{Fe}$ IV in the ground $3 d^{5}{ }^{6} S$ state is very similar in magnitude and features to this total $\sigma_{\mathrm{PI}}[10]$. The circles in the figure correspond to the results from the central-field approximation [13] which, when extrapolated to lower energies, overestimate the cross section by about five times higher than the CC cross section (present astrophysical models employ the central-field cross sections shown, and are thus likely to overestimate the photoionization rates of Fe III). We present Fig. 2 to emphasize that the new calculations are for both photoionization and recombination of Fe III, thereby providing a complete and consistent set of data to enable accurate calculations for ionization balance under photoionization equilibrium. The ionization balance equation [14]

$$
N_{X^{+}} \int \frac{4 \pi J_{\nu}}{h \nu} \sigma_{\mathrm{PI}} d \nu=N_{e} N_{X^{2}+\alpha_{R}}
$$

requires the total photoionization cross section of the ground state, and the total recombination rate coefficient as presented in this paper.

\section{B. High- $n$ states: Collision strengths for dielectronic recombination}

For the high- $n$ states $\left(n_{\max } \leqslant n \leqslant \infty\right)$ of group (B) the collision strengths for dielectronic recombination, $\Omega(\mathrm{DR})$, are obtained in the CC approximation employing Bell and

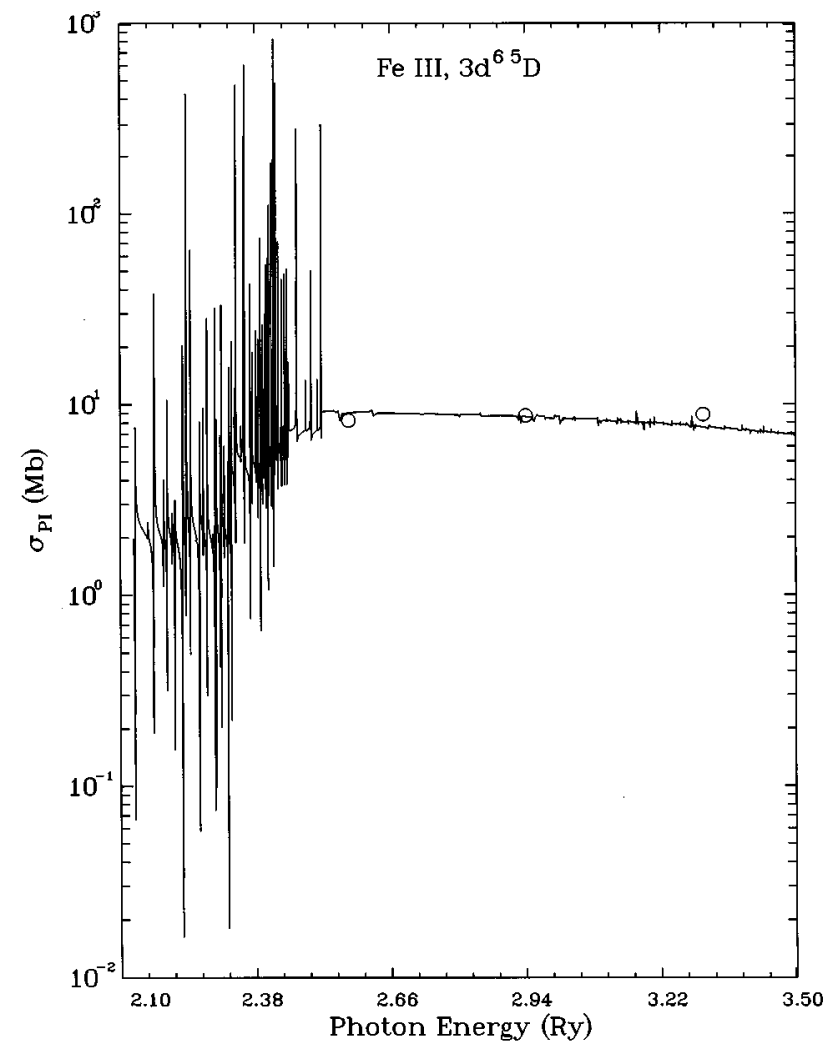

FIG. 2. Total photoionization cross section of the $3 d^{65} D$ ground state of Fe III in the 49-CC calculations. The circles correspond to central-field values [13].

Seaton theory for DR $[9,2]$. In the energy region of these states below each target state, the electron-electron scattering matrix $\mathscr{S}_{e e}$ is partitioned into submatrices, $\chi_{o o}, \chi_{o c}, \chi_{c o}$, $\chi_{c c}$, where $o$ denotes the open and $c$ denotes the closed channels. Diagonalizing the $\chi_{c c}$ with the diagonalizing matrix $\mathbf{N}$, we define $\boldsymbol{\chi}_{c c} \mathbf{N}=\mathbf{N} \chi_{c c}, \quad \chi_{o c}^{\prime}=\chi_{o c} \mathbf{N}, \quad$ and $\chi_{c o}^{\prime}=\mathbf{N}^{T} \chi_{c o}$. From the unitarity condition of the general scattering matrix, which includes the radiation damping, the DR probability $P_{\alpha}$ for the entrance channel $\alpha$ can be written in terms of the matrix elements as

$$
\begin{aligned}
P_{\alpha}= & G(\nu) \sum_{\gamma}\left\{\left(\sum_{\gamma^{\prime}} \chi_{\alpha \gamma^{\prime}}^{\prime} \mathbf{N}_{\gamma \gamma^{\prime}}\right)\left[\frac{1}{\boldsymbol{\chi}_{\gamma \gamma^{\prime}}-g(\nu) \exp (-2 \pi i \nu)}\right]\right. \\
& \left.\times\left[\frac{1}{\boldsymbol{\chi}_{\gamma \gamma}^{*}-g(\nu) \exp (+2 \pi i \nu)}\right]\left(\sum_{\gamma^{\prime}} \chi_{\gamma^{\prime} \alpha}^{\prime *} \mathbf{N}_{\gamma \gamma^{\prime}}^{*}\right)\right\},(2.8)
\end{aligned}
$$

where $g(\nu)=\exp \left(\pi \nu^{3} A_{r} / z^{2}\right), G(\nu)=g(\nu)^{2}-1, A_{r}$ is the sum of the radiative decay probabilities for the available decay routes from a given excited state of the target ion, $\nu$ is the effective quantum number associated with the resonance series, $z$ is the ion charge, and the summations go over the closed channels $\gamma \gamma^{\prime}$ contributing to DR. The DR collision strength $\Omega(\mathrm{DR})$ is then obtained as

$$
\Omega(\mathrm{DR})=\sum_{S L \pi} \sum_{\alpha} \frac{1}{2}(2 S+1)(2 L+1) P_{\alpha}^{S L \pi} .
$$




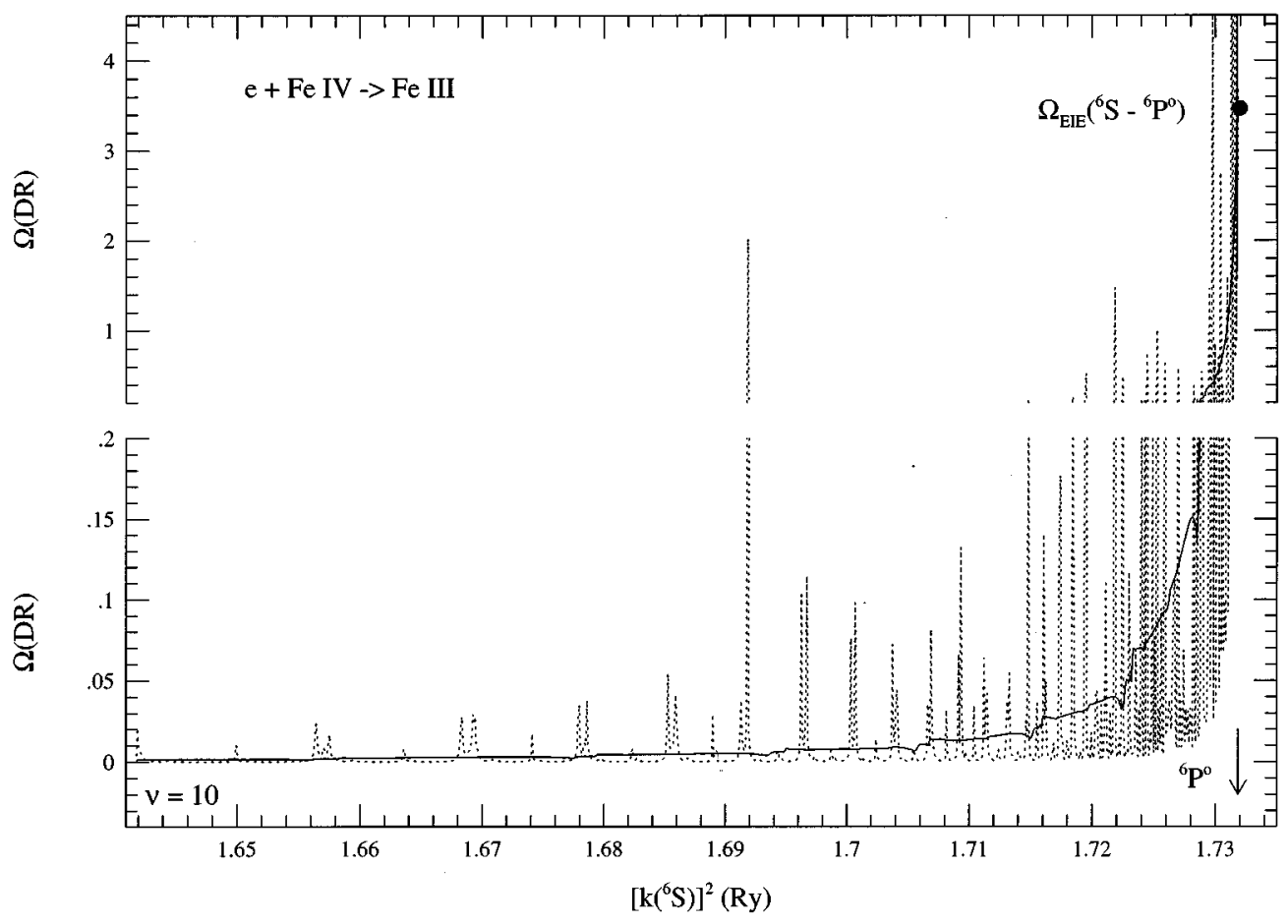

FIG. 3. DR collision strength $\Omega(\mathrm{DR})$ for the process $e+\mathrm{Fe} \mathrm{IV}$ $\rightarrow$ Fe III: (i) detailed with autoionizing resonances (dotted curves) and (ii) resonance averaged (solid curves). DR calculations start at effective quantum number $\nu=10.0$ and the arrow points out the dipole allowed core state ${ }^{6} P^{o}$. The filled circle corresponds to the value, 3.47, of excitation collision strength, $\Omega(\mathrm{EIE})$, for the transition ${ }^{6} S-{ }^{6} P^{o}$.
Averaging over the resonances of DR, the DR probability can be written as

$$
\begin{aligned}
\left\langle P_{\alpha}\right\rangle= & G(\nu)\left[\sum_{\gamma} \frac{\left|\chi_{\alpha \gamma}^{\prime}\right|^{2} \sum_{i}\left|\mathbf{N}_{\mathbf{i} \gamma}\right|^{2}}{G(\nu)+1-\left|\boldsymbol{\chi}_{\gamma \gamma}\right|^{2}}\right. \\
& \left.+2 \sum_{\gamma \neq \gamma^{\prime}} \operatorname{Re}\left(\frac{\chi_{\alpha \gamma}^{\prime} \chi_{\gamma^{\prime} \alpha}^{\prime} \sum_{i} \mathbf{N}_{i \gamma} \mathbf{N}_{i \gamma^{\prime}}^{*}}{G(\nu)+1-\boldsymbol{\chi}_{\gamma \gamma} \boldsymbol{\chi}_{\gamma^{\prime} \gamma^{\prime}}}\right)\right],
\end{aligned}
$$

where the summation over $i$ goes through all the closed channels. We are interested mainly in the channels linked to the ground state of the target ion and hence in the DR cross sections for the transitions from excited states that are linked radiatively to the ground state. For the Fe IV target, we have only one state, $3 d^{4}{ }^{5} D 4 p\left({ }^{6} P^{o}\right)$, that is dipole allowed to the ground $3 d^{56} S$ state. The radiative transition probability for the transition ${ }^{6} S \rightarrow{ }^{6} P^{o}$ is 0.771 nsec, obtained from the OP $f$ value of 0.407 for this transition [15].

The DR collision strengths for $e+\mathrm{Fe}$ IV $\rightarrow$ Fe III are obtained in both forms, detailed with autoionizing resonances and averaged over the resonances. Figure 3 presents $\Omega(\mathrm{DR})$ in both forms, the detailed form (dotted curve) and the resonance averaged form (solid curve). The computation for $\Omega(\mathrm{DR})$ starts at $\nu=10.0$, as specified in the figure, where the collision strength is almost zero. As the energy approaches the excited threshold ${ }^{6} P^{o}$ state of the target, the autoionizing resonances of DR get narrower and denser while the background rises as seen in the figure. The sharp rising of $\Omega(D R)$ near the excited threshold is clear in the solid curve for the averaged collision strength which peaks at 3.46 exactly at the ${ }^{6} P^{o}$ state. Some small features can be noticed in the solid curve. Since the averaging of resonances is carried out only to the ones that belong to Rydberg series of the threshold ${ }^{6} P^{o}$, the crookedness in the curve is due to the interference effects of the resonances from higher thresholds, beyond ${ }^{6} P^{o}$. These introduce a small uncertainty [3] in $\alpha_{R}$. The $\alpha_{R}$ values reported here correspond to resonance averaged values of $\Omega(D R)$, since these values are more stable numerically. The difference between the DR rates obtained from the detailed collision strengths and the averaged ones for the present ion is $8 \%$, indicating the energy mesh is good enough for resolving the resonances.

The filled circle in Fig. 3 corresponds to the value of $\Omega_{\mathrm{EIE}}\left({ }^{6} S-{ }^{6} P^{o}\right)$, the collision strength for electron impact excitation (EIE) of the ground state ${ }^{6} S$ to the excited ${ }^{6} P^{o}$ state. The value of $\Omega_{\mathrm{EIE}}\left({ }^{6} S-{ }^{6} P^{o}\right)$ is 3.47 , about the same as the $\langle\Omega(\mathrm{DR})\rangle$ peak value of 3.46 mentioned above, and is obtained independently from a close coupling scattering calculation for the ion employing the same 49-state expansion. This provides a check to the peak value of $\Omega(\mathrm{DR})$ at the threshold satisfying the conservation condition of the scattering flux

$$
\lim _{n \rightarrow \infty}\langle\Omega(n ; D R)\rangle=\lim _{k^{2} \rightarrow 0} \Omega\left(k^{2} ; \mathrm{EIE}\right)
$$

such that the trapped flux due to DR right below the threshold should be equal to that released due to excitation of the target. The other check that we carry out is for the effects of multipole potentials on the $\Omega(\mathrm{DR})$. We calculate independently, using the same wave function expansion, the values of $\Omega$ (EIE) at the threshold with inclusion of multipole potentials and without them. A small discrepancy between the two values is an indicator of convergence of the wave function expansion and negligible effects of these potentials on the scattering quantities. A proper choice of the $R$-matrix boundary and $R$-matrix basis set can achieve this goal [2]. 


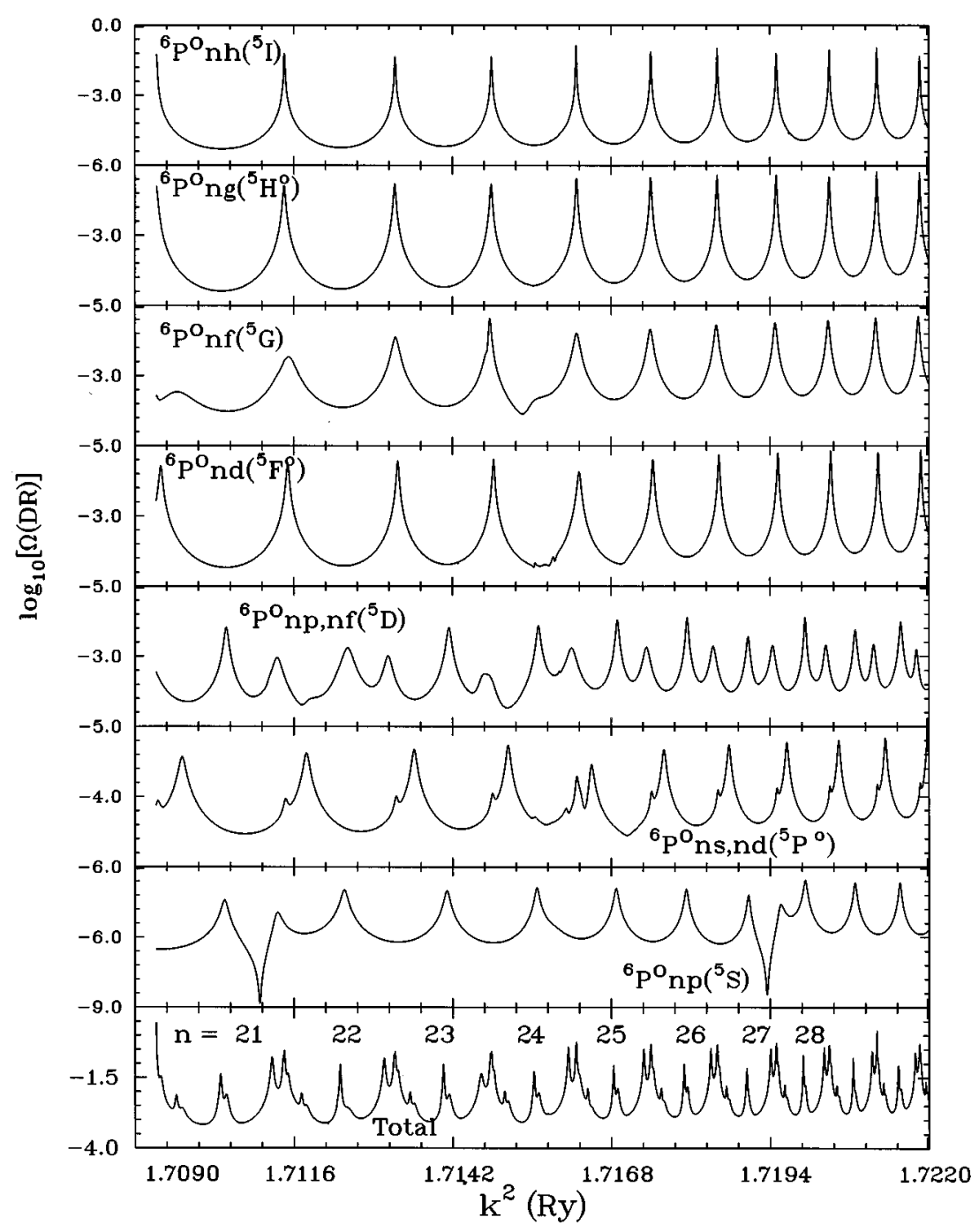

FIG. 4. Detailed analysis of the photon spectrum, in the process $e+$ Fe IV $\rightarrow$ Fe III, in the small energy range from 1.71 to $1.722 \mathrm{Ry}$ of the electron corresponding to the range from $\nu=20$ to about 30 of the Rydberg series belonging to ${ }^{6} P^{o} \nu \ell$. The bottom panel presents total $\Omega(\mathrm{DR})$ while the upper panels show the individual contributions of the symmetries ${ }^{5} S,{ }^{5} P^{o}$, ${ }^{5} D$, etc. as specified in each panel. The $n$ complexes are designated. The individual patterns are identified as ${ }^{6} P^{o} n p\left({ }^{5} S\right), \quad{ }^{6} P^{o} n s, n d\left({ }^{5} P^{o}\right), \quad{ }^{6} P^{o} n p, n f\left({ }^{5} D\right)$, etc.
For the present case of ( $e, \mathrm{Fe}$ IV) scattering, the values of $\Omega(\mathrm{EIE})$ with and without the multipole potential contributions are 3.70 and 3.76 .

Figure 4 presents a detailed analysis of the photon spectrum which appears as autoionizing resonance lines in Fig. 3. The structures are resolved in a very small energy range from 1.71 to 1.722 Ry of the electron corresponding to effective quantum number ranging from $\nu=20$ to about 30 of the Rydberg series of states belonging to the ${ }^{6} P^{o}$ state. The bottom panel corresponds to total $\Omega(\mathrm{DR})$ while the upper ones correspond to individual contributions of the first seven symmetries ${ }^{5} S,{ }^{5} P^{o},{ }^{5} D$, etc. as specified in the panels. The pattern of each $n$ complex, which repeats itself but gets more compressed and rises slowly with increase of energy, is distinct for the total and for each individual symmetry. The relevant Rydberg series for each pattern has been identified through quantum defects at the peaks of the resonance series and is specified in each panel: ${ }^{6} P^{o} n p\left({ }^{5} S\right),{ }^{6} P^{o} n s, n d\left({ }^{5} P^{o}\right)$, ${ }^{6} P^{o} n p, n f\left({ }^{5} D\right)$, etc. The third panel from the bottom shows the interference of two Rydberg series, ${ }^{6} P^{o} n s\left({ }^{5} P^{o}\right)$ and ${ }^{6} P^{o} n d\left({ }^{5} P^{o}\right)$, the first resonance peaks belonging to the $d$ series, which is narrower than the $s$ series. Such interferences of the series will introduce errors in the treatment of isolated resonance approximation for the recombination rate coeffi- cients. A few isolated structures in the patterns, such as two down peaks in the first panel and split of one $s$ resonance in the third panel, are due to interference with some resonances belonging to higher states, lying above the ${ }^{6} P^{o}$ state, of Fe IV.

At very low electron energies and temperatures the recombination process is also dominated by recombination to very high- $n$ states just below the ground state of the recombining ion (as distinct from the high- $n$ recombination via resonances belonging to excited states, i.e., DR, as described above). These contributions are included in the hydrogenic approximation; it is found necessary to calculate explicitly recombination contributions at low temperatures to states with up to $n=800$. Although the recombination to high- $n$ states is dominated by DR at higher temperatures, the radiative part involving the photoionization cross section of high$n$ states is "topped up" with the hydrogenic recombination rate coefficient. For an ion with charge $z$, we have the $z$-scaled formula $\alpha_{R}(z, T)=\alpha_{R}\left(1, T / z^{2}\right)$, in terms of the recombination rate coefficient for neutral hydrogen [14]. We calculate the hydrogenic recombination rate coefficients $\alpha_{R}(z, T)$ for states $n=10-800$ employing photoionization cross sections of hydrogen obtained using the FORTRAN program by Storey and Hummer [16], and for states $n=801-$ $\infty$ using the sum [17] 
TABLE I. Total recombination rate coefficients $\alpha_{R}(T)$ in units of $\mathrm{cm}^{3} \mathrm{~s}^{-1}$, for $e+\mathrm{Fe} \mathrm{IV} \rightarrow \mathrm{Fe}$ III for a wide range of temperatures $T(\mathrm{~K})$. A value of $a[-b]$ for $\alpha_{R}$ means $a \times 10^{-b}$.

\begin{tabular}{|c|c|c|c|c|c|}
\hline $\log _{10} T$ & $\alpha_{R}$ & $\log _{10} T$ & $\alpha_{R}$ & $\log _{10} T$ & $\alpha_{R}$ \\
\hline 1.0 & $4.20[-10]$ & 3.7 & $6.86[-12]$ & 6.4 & $3.08[-13]$ \\
\hline 1.1 & $3.67[-10]$ & 3.8 & $6.11[-12]$ & 6.5 & $2.23[-13]$ \\
\hline 1.2 & $3.22[-10]$ & 3.9 & $5.54[-12]$ & 6.6 & $1.64[-13]$ \\
\hline 1.3 & $2.82[-10]$ & 4.0 & $5.08[-12]$ & 6.7 & $1.21[-13]$ \\
\hline 1.4 & $2.47[-10]$ & 4.1 & $4.66[-12]$ & 6.8 & $8.86[-14]$ \\
\hline 1.5 & $2.16[-10]$ & 4.2 & $4.26[-12]$ & 6.9 & $6.50[-14]$ \\
\hline 1.6 & $1.89[-10]$ & 4.3 & $3.84[-12]$ & 7.0 & $4.76[-14]$ \\
\hline 1.7 & $1.65[-10]$ & 4.4 & $3.43[-12]$ & 7.1 & $3.48[-14]$ \\
\hline 1.8 & $1.44[-10]$ & 4.5 & $3.05[-12]$ & 7.2 & $2.55[-14]$ \\
\hline 1.9 & $1.25[-10]$ & 4.6 & $2.78[-12]$ & 7.3 & $1.86[-14]$ \\
\hline 2.0 & $1.08[-10]$ & 4.7 & $2.70[-12]$ & 7.4 & $1.36[-14]$ \\
\hline 2.1 & $9.30[-11]$ & 4.8 & $2.85[-12]$ & 7.5 & $9.96[-15]$ \\
\hline 2.2 & $7.99[-11]$ & 4.9 & $3.19[-12]$ & 7.6 & $7.28[-15]$ \\
\hline 2.3 & $6.84[-11]$ & 5.0 & $3.58[-12]$ & 7.7 & $5.33[-15]$ \\
\hline 2.4 & $5.83[-11]$ & 5.1 & $3.85[-12]$ & 7.8 & $3.90[-15]$ \\
\hline 2.5 & $4.95[-11]$ & 5.2 & $3.91[-12]$ & 7.9 & $2.86[-15]$ \\
\hline 2.6 & $4.19[-11]$ & 5.3 & $3.76[-12]$ & 8.0 & $2.10[-15]$ \\
\hline 2.7 & $3.54[-11]$ & 5.4 & $3.42[-12]$ & 8.1 & $1.54[-15]$ \\
\hline 2.8 & $2.98[-11]$ & 5.5 & $2.97[-12]$ & 8.2 & $1.14[-15]$ \\
\hline 2.9 & $2.50[-11]$ & 5.6 & $2.50[-12]$ & 8.3 & $8.40[-16]$ \\
\hline 3.0 & $2.10[-11]$ & 5.7 & $2.03[-12]$ & 8.4 & $6.24[-16]$ \\
\hline 3.1 & $1.77[-11]$ & 5.8 & $1.61[-12]$ & 8.5 & $4.65[-16]$ \\
\hline 3.2 & $1.49[-11]$ & 5.9 & $1.26[-12]$ & 8.6 & $3.48[-16]$ \\
\hline 3.3 & $1.26[-11]$ & 6.0 & $9.65[-13]$ & 8.7 & $2.63[-16]$ \\
\hline 3.4 & $1.07[-11]$ & 6.1 & $7.33[-13]$ & 8.8 & $1.99[-16]$ \\
\hline 3.5 & $9.14[-12]$ & 6.2 & $5.52[-13]$ & 8.9 & $1.53[-16]$ \\
\hline 3.6 & $7.86[-12]$ & 6.3 & $4.13[-13]$ & 9.0 & $1.18[-16]$ \\
\hline
\end{tabular}

$$
\Delta(n)=\alpha_{n}\left(\frac{n}{n+1}\right)^{3}\left(\frac{1+n}{2}\right) .
$$

We find for Fe III, contrary to most other ions [18], that this top-up hydrogenic part provides significant contribution to the total $\alpha_{R}$, about $50 \%$ up to $T=400 \mathrm{~K}$, then decaying slowly to $24 \%$ at $T=10000 \mathrm{~K}$.

\section{RESULTS AND DISCUSSIONS}

Total recombination rate coefficients $\alpha_{R}(T)$ for the recombination process, $e+\mathrm{Fe}$ IV $\rightarrow$ Fe III, are obtained over a wide range of temperatures $1.0 \leqslant \log _{10} T \leqslant 9.0$, with $\Delta \log _{10} T=0.1$. The values of $\alpha_{R}(T)$ are given in Table I and plotted in Fig. 5 (the solid curve). The curve shows the standard pattern of the total recombination rate coefficients [3]. At low temperatures, as $T$ increases, the rate decreases until it reaches the temperature region where DR begins to dominate, culminating in the high- $T$ DR bump, whereupon the rate decreases smoothly. However, before the high- $T$ DR bump at $\log _{10} T=5.2$, there exists a small but distinct low$T$ bump due to the low- $n$ autoionizing resonances in the near-threshold regions of the Fe III photoionization cross sections.

Comparison of the present total $\alpha_{R}$ (solid curve) is made with the RR rates (dashed curve) and the DR rates (chaindashed curve) of Woods et al. [4]. Their total rates can be

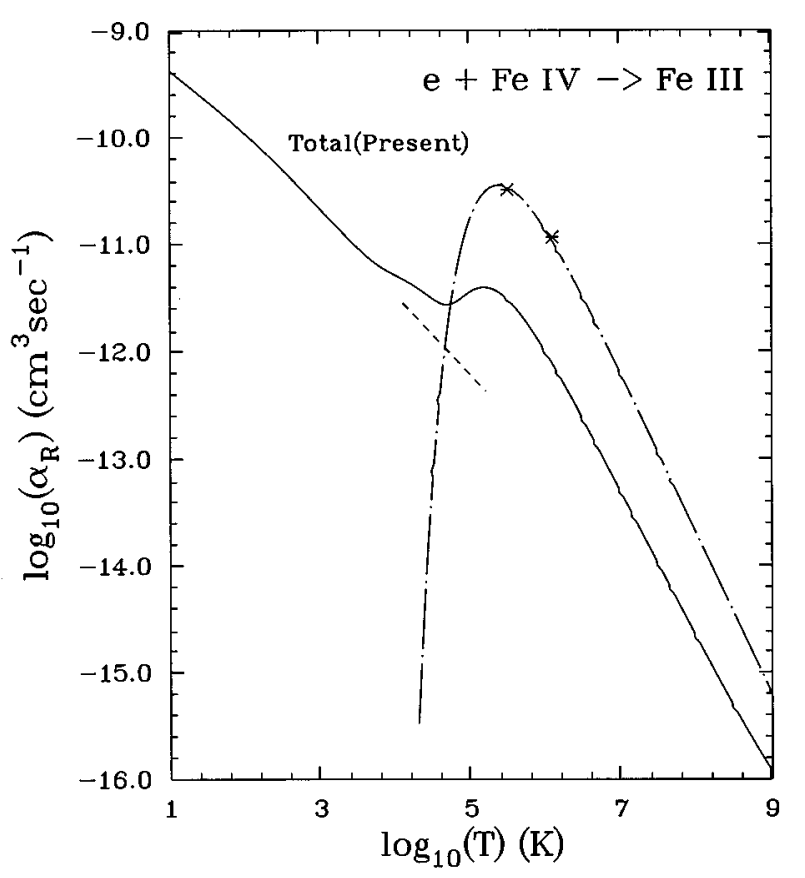

FIG. 5. Total recombination rate coefficients $\alpha_{R}(T)$ for the recombined ion Fe III of the present work (solid curve). The dashed curve corresponds to RR rates and the chain-dashed curve corresponds to DR rates of Ref. [4] and the asterisks correspond to DR rates of Ref. [7]. 
TABLE II. Recombination rate coefficients (in units of $\mathrm{cm}^{3} \mathrm{~s}^{-1}$ ) at four temperatures, $T=100,1000,5012$, and $10000 \mathrm{~K}$, of the individual dominant contributing states from quintet and septet symmetries. A value of $a[-b]$ for $\alpha_{R}$ means $a \times 10^{-b}$.

\begin{tabular}{|c|c|c|c|c|c|c|c|c|c|c|c|}
\hline \multicolumn{3}{|c|}{$100 \mathrm{~K}$} & \multicolumn{3}{|c|}{$1000 \mathrm{~K}$} & \multicolumn{3}{|c|}{$5012 \mathrm{~K}$} & \multicolumn{3}{|c|}{$10000 \mathrm{~K}$} \\
\hline State & & $\alpha_{R}$ & State & & $\alpha_{R}$ & State & & $\alpha_{R}$ & State & & $\alpha_{R}$ \\
\hline $3 d^{5}{ }^{4} F^{e} 4 p$ & ${ }^{5} D^{o}$ & $6.99[-12]$ & $3 d^{6}$ & ${ }^{5} D^{e}$ & $1.98[-12]$ & $3 d^{6}$ & ${ }^{5} D^{e}$ & $1.06[-12]$ & $3 d^{6}$ & ${ }^{5} D^{e}$ & $9.08[-13]$ \\
\hline $3 d^{54} F^{e} 5 p$ & ${ }^{5} D^{o}$ & $5.18[-12]$ & $3 d^{54} F^{e} 4 p$ & ${ }^{5} D^{o}$ & $8.64[-13]$ & $3 d^{5}{ }^{4} D^{e} 4 p$ & ${ }^{5} D^{o}$ & $1.14[-13]$ & $3 d^{5}{ }^{6} S^{e} 4 p$ & ${ }^{5} P^{o}$ & $1.29[-13]$ \\
\hline $3 d^{6}$ & ${ }^{5} D^{e}$ & $4.40[-12]$ & $3 d^{5}{ }^{4} F^{e} 5 p$ & ${ }^{5} D^{o}$ & $6.20[-13]$ & $3 d^{5}{ }^{6} S^{e} 4 p$ & ${ }^{5} P^{o}$ & $1.11[-13]$ & $3 d^{5}{ }^{4} D^{e} 4 p$ & ${ }^{5} D^{o}$ & $7.56[-14]$ \\
\hline $3 d^{5}{ }^{4} P^{e} 4 p$ & ${ }^{5} P^{o}$ & $4.16[-12]$ & $3 d^{5}{ }^{4} P^{e} 4 p$ & ${ }^{5} P^{o}$ & $4.57[-13]$ & $3 d^{5}{ }^{4} F^{e} 4 p$ & ${ }^{5} D^{o}$ & $1.10[-13]$ & $3 d^{54} D^{e} 4 p$ & ${ }^{5} F^{o}$ & $7.27[-14]$ \\
\hline $3 d^{54} P^{e} 5 p$ & ${ }^{5} P^{o}$ & $3.20[-12]$ & $3 d^{54} F^{e} 4 p$ & ${ }^{5} F^{o}$ & $3.55[-13]$ & $3 d^{5}{ }^{4} D^{e} 4 p$ & ${ }^{5} F^{o}$ & $9.23[-14]$ & $3 d^{54} G^{e} 4 p$ & ${ }^{5} H^{o}$ & $6.99[-14]$ \\
\hline $3 d^{5}{ }^{4} F^{e} 4 p$ & ${ }^{5} F^{o}$ & $2.99[-12]$ & $3 d^{54} P^{e} 5 p$ & ${ }^{5} P^{o}$ & $3.52[-13]$ & $3 d^{5}{ }^{4} P^{e} 4 p$ & ${ }^{5} P^{o}$ & $8.72[-14]$ & $3 d^{5}{ }^{4} P^{e} 4 p$ & ${ }^{5} P^{o}$ & $5.55[-14]$ \\
\hline $3 d^{54} F^{e} 5 p$ & ${ }^{5} F^{o}$ & $2.81[-12]$ & $3 d^{54} F^{e} 5 p$ & ${ }^{5} F^{o}$ & $3.35[-13]$ & $3 d^{5}{ }^{4} F^{e} 5 p$ & ${ }^{5} D^{o}$ & $7.72[-14]$ & $3 d^{4} 4 s^{6} D^{e} 4 p$ & ${ }^{5} P^{o}$ & $5.54[-14]$ \\
\hline $3 d^{54} P^{e} 6 p$ & ${ }^{5} P^{o}$ & $2.80[-12]$ & $3 d^{5}{ }^{4} G^{e} 4 p$ & ${ }^{5} F^{o}$ & $3.23[-13]$ & $3 d^{5}{ }^{6} S^{e} 4 f$ & ${ }^{5} F^{o}$ & $7.01[-14]$ & $3 d^{4} 4 s^{6} D^{e} 4 p$ & ${ }^{5} F^{o}$ & $5.21[-14]$ \\
\hline $3 d^{54} G^{e} 4 p$ & ${ }^{5} F^{o}$ & $2.42[-12]$ & $3 d^{5}{ }^{4} P^{e} 6 p$ & ${ }^{5} P^{o}$ & $2.96[-13]$ & $3 d^{5}{ }^{4} D^{e} 4 p$ & ${ }^{5} P^{o}$ & $6.89[-14]$ & $3 d^{5}{ }^{4} G^{e} 4 p$ & ${ }^{5} F^{o}$ & $4.84[-14]$ \\
\hline $3 d^{5}{ }^{4} D^{e} 4 p$ & ${ }^{5} D^{o}$ & $1.32[-12]$ & $3 d^{5}{ }^{6} S^{e} 4 p$ & ${ }^{5} P^{o}$ & $2.32[-13]$ & $3 d^{5}{ }^{4} D^{e} 5 p$ & ${ }^{5} D^{o}$ & $6.76[-14]$ & $3 d^{5}{ }^{4} F^{e} 4 p$ & ${ }^{5} D^{o}$ & $4.83[-14]$ \\
\hline $3 d^{5}{ }^{4} P^{e} 6 p$ & ${ }^{5} D^{o}$ & $1.13[-12]$ & $3 d^{5}{ }^{4} D^{e} 4 p$ & ${ }^{5} D^{o}$ & $1.89[-13]$ & $3 d^{5}{ }^{4} G^{e} 4 p$ & ${ }^{5} F^{o}$ & $6.62[-14]$ & $3 d^{5}{ }^{6} S^{e} 4 f$ & ${ }^{5} F^{o}$ & $4.60[-14]$ \\
\hline $3 d^{5}{ }^{6} S^{e} 4 p$ & ${ }^{5} P^{o}$ & $1.03[-12]$ & $3 d^{5}{ }^{6} S^{e} 4 f$ & ${ }^{5} F^{o}$ & $1.71[-13]$ & $3 d^{5}{ }^{6} S^{e} 5 f$ & ${ }^{5} F^{o}$ & $6.18[-14]$ & $3 d^{54} D^{e} 5 p$ & ${ }^{5} D^{o}$ & $4.24[-14]$ \\
\hline $3 d^{54} P^{e} 5 p$ & ${ }^{5} D^{o}$ & $1.01[-12]$ & $3 d^{5}{ }^{6} S^{e} 5 f$ & ${ }^{5} F^{o}$ & $1.51[-13]$ & $3 d^{5}{ }^{4} D^{e} 6 p$ & ${ }^{5} D^{o}$ & $6.07[-14]$ & $3 d^{5}{ }^{4} D^{e} 4 p$ & ${ }^{5} P^{o}$ & $4.24[-14]$ \\
\hline $3 d^{54} G^{e} 5 p$ & ${ }^{5} F^{o}$ & $7.02[-13]$ & $3 d^{5}{ }^{6} S^{e} 5 p$ & ${ }^{5} P^{o}$ & $1.37[-13]$ & $3 d^{5}{ }^{4} D^{e} 5 p$ & ${ }^{5} F^{o}$ & $5.12[-14]$ & $3 d^{5}{ }^{6} S^{e} 5 f$ & ${ }^{5} F^{o}$ & $4.04[-14]$ \\
\hline $3 d^{5}{ }^{6} S^{e} 4 f$ & ${ }^{5} F^{o}$ & $5.60[-13]$ & $3 d^{5}{ }^{4} P^{e} 6 p$ & ${ }^{5} D^{o}$ & $1.24[-13]$ & $3 d^{5}{ }^{4} D^{e} 5 p$ & ${ }^{5} P^{o}$ & $5.11[-14]$ & $3 d^{54} D^{e} 6 p$ & ${ }^{5} D^{o}$ & $3.83[-14]$ \\
\hline $3 d^{5}{ }^{6} S^{e} 5 p$ & ${ }^{5} P^{o}$ & $5.06[-13]$ & $3 d^{5}{ }^{6} S^{e} 6 f$ & ${ }^{5} F^{o}$ & $1.24[-13]$ & $3 d^{5}{ }^{4} P^{e} 5 p$ & ${ }^{5} P^{o}$ & $4.98[-14]$ & $3 d^{54} F^{e} 5 p$ & ${ }^{5} D^{o}$ & $3.42[-14]$ \\
\hline $3 d^{54} P^{e} 4 p$ & ${ }^{5} D^{o}$ & $5.06[-13]$ & $3 d^{5}{ }^{4} P^{e} 5 p$ & ${ }^{5} D^{o}$ & $1.14[-13]$ & $3 d^{5}{ }^{4} F^{e} 4 p$ & ${ }^{5} F^{o}$ & $4.90[-14]$ & $3 d^{5}{ }^{4} G^{e} 4 f$ & ${ }^{5} P^{o}$ & $3.40[-14]$ \\
\hline $3 d^{5}{ }^{6} S^{e} 5 f$ & ${ }^{5} F^{o}$ & $4.96[-13]$ & $3 d^{5}{ }^{4} G^{e} 5 p$ & ${ }^{5} F^{o}$ & $9.69[-14]$ & $3 d^{5}{ }^{6} S^{e} 6 f$ & ${ }^{5} F^{o}$ & $4.55[-14]$ & $3 d^{5}{ }^{4} D^{e} 5 p$ & ${ }^{5} F^{o}$ & $3.13[-14]$ \\
\hline $3 d^{5}{ }^{6} S^{e} 6 f$ & ${ }^{5} F^{o}$ & $4.78[-13]$ & $3 d^{5}{ }^{6} S^{e} 6 g$ & ${ }^{5} G^{e}$ & $8.63[-14]$ & $3 d^{5}{ }^{6} S^{e} 5 p$ & ${ }^{5} P^{o}$ & $4.34[-14]$ & $3 d^{54} D^{e} 5 p$ & ${ }^{5} P^{o}$ & $3.07[-14]$ \\
\hline $3 d^{5}{ }^{4} D^{e} 4 p$ & ${ }^{5} P^{o}$ & $3.78[-13]$ & $3 d^{5}{ }^{4} D^{e} 4 p$ & ${ }^{5} P^{o}$ & $7.67[-14]$ & $3 d^{5}{ }^{4} F^{e} 5 p$ & ${ }^{5} F^{o}$ & $4.17[-14]$ & $3 d^{5}{ }^{4} P^{e} 4 p$ & ${ }^{5} D^{o}$ & $3.03[-14]$ \\
\hline Sum: & & $4.31[-11]$ & & & $7.08[-12]$ & & & $2.38[-$ & & & $1.89[-12]$ \\
\hline Total $=$ & & $1.08[-10]$ & & & $2.10[-11]$ & & & $6.86[-12]$ & & & $5.08[-12]$ \\
\hline$\%$ contribution & & $40 \%$ & & & $34 \%$ & & & $35 \%$ & & & $37 \%$ \\
\hline $3 d^{5}{ }^{6} S^{e} 5 f$ & ${ }^{7} F^{o}$ & $6.96[-$ & $3 d^{5}{ }^{6} S^{e} 5 f$ & ${ }^{7} F^{o}$ & $\mathrm{~L}$ & $3 d^{5}{ }^{6} S^{e} 4 p$ & ${ }^{7} P^{o}$ & 3] & $3 d^{5}{ }^{6} S^{e} 4 p$ & ${ }^{7} P^{o}$ & 2.25 \\
\hline $3 d^{5}{ }^{6} S^{e} 6 f$ & ${ }^{7} F^{o}$ & $5.08[-13]$ & $3 d^{5}{ }^{6} S^{e} 6 f$ & ${ }^{7} F^{o}$ & $1.58[-13]$ & $3 d^{5}{ }^{6} S^{e} 4 f$ & ${ }^{7} F^{o}$ & $9.67[-14]$ & $3 d^{5}{ }^{6} S^{e} 4 f$ & ${ }^{7} F^{o}$ & $1.63[-13]$ \\
\hline $3 d^{5}{ }^{6} S^{e} 4 f$ & ${ }^{7} F^{o}$ & $4.45[-13]$ & $3 d^{5}{ }^{6} S^{e} 4 f$ & ${ }^{7} F^{o}$ & $1.40[-13]$ & $3 d^{5}{ }^{6} S^{e} 5 f$ & ${ }^{7} F^{o}$ & $9.05[-14]$ & $3 d^{4} 4 s^{6} D^{e} 4 p$ & ${ }^{7} P^{o}$ & $1.45[-13]$ \\
\hline $3 d^{5}{ }^{6} S^{e} 6 g$ & ${ }^{7} G^{e}$ & $3.98[-13]$ & $3 d^{5}{ }^{6} S^{e} 6 g$ & ${ }^{7} G^{e}$ & $1.22[-13]$ & $3 d^{4} 4 s^{6} D^{e} 4 p$ & ${ }^{7} F^{o}$ & $7.05[-14]$ & $3 d^{4} 4 s^{6} D^{e} 4 p$ & ${ }^{7} F^{o}$ & $1.06[-13]$ \\
\hline $3 d^{4} 4 s^{6} D^{e} 4 p$ & ${ }^{7} F^{o}$ & $3.93[-13]$ & $3 d^{4} 4 s^{6} D^{e} 4 p$ & ${ }^{7} F^{o}$ & $1.22[-13]$ & $3 d^{5}{ }^{6} S^{e} 6 f$ & ${ }^{7} F^{o}$ & $6.63[-14]$ & $3 d^{5}{ }^{6} S^{e} 5 f$ & ${ }^{7} F^{o}$ & $5.96[-14]$ \\
\hline $3 d^{5}{ }^{6} S^{e} 4 p$ & ${ }^{7} P^{o}$ & $3.72[-13]$ & $3 d^{5}{ }^{6} S^{e} 4 p$ & ${ }^{7} P^{o}$ & $1.16[-13]$ & $3 d^{4} 4 s^{6} D^{e} 4 p$ & ${ }^{7} P^{o}$ & $5.09[-14]$ & $3 d^{5}{ }^{6} S^{e} 6 f$ & ${ }^{7} F^{o}$ & $4.37[-14]$ \\
\hline $3 d^{5}{ }^{6} S^{e} 7 f$ & ${ }^{7} F^{o}$ & $3.62[-13]$ & $3 d^{5}{ }^{6} S^{e} 7 f$ & ${ }^{7} F^{o}$ & $1.13[-13]$ & $3 d^{5}{ }^{6} S^{e} 6 g$ & ${ }^{7} G^{e}$ & $4.79[-14]$ & $3 d^{5}{ }^{6} S^{e} 7 f$ & ${ }^{7} F^{o}$ & $3.13[-14]$ \\
\hline $3 d^{5}{ }^{6} S^{e} 7 g$ & ${ }^{7} G^{e}$ & $3.51[-13]$ & $3 d^{5}{ }^{6} S^{e} 7 g$ & ${ }^{7} G^{e}$ & $1.08[-13]$ & $3 d^{5}{ }^{6} S^{e} 7 f$ & ${ }^{7} F^{o}$ & $4.74[-14]$ & $3 d^{5}{ }^{6} S^{e} 6 g$ & ${ }^{7} G^{e}$ & $2.97[-14]$ \\
\hline $3 d^{5}{ }^{6} S^{e} 5 g$ & ${ }^{7} G^{e}$ & $3.46[-13]$ & $3 d^{5}{ }^{6} S^{e} 5 g$ & ${ }^{7} G^{e}$ & $1.06[-13]$ & $3 d^{5}{ }^{6} S^{e} 7 g$ & ${ }^{7} G^{e}$ & $4.23[-14]$ & $3 d^{4} 4 s^{6} D^{e} 4 p$ & ${ }^{7} D^{o}$ & $2.81[-14]$ \\
\hline $3 d^{5}{ }^{6} S^{e} 5 p$ & ${ }^{7} P^{o}$ & $2.93[-13]$ & $3 d^{5}{ }^{6} S^{e} 5 p$ & ${ }^{7} P^{o}$ & $9.16[-14]$ & $3 d^{5}{ }^{6} S^{e} 5 g$ & ${ }^{7} G^{e}$ & $4.16[-14]$ & $3 d^{5}{ }^{6} S^{e} 5 p$ & ${ }^{7} P^{o}$ & $2.71[-14]$ \\
\hline $3 d^{5}{ }^{6} S^{e} 8 g$ & ${ }^{7} G^{e}$ & $2.88[-13]$ & $3 d^{5}{ }^{6} S^{e} 8 g$ & ${ }^{7} G^{e}$ & $8.84[-14]$ & $3 d^{5}{ }^{6} S^{e} 5 p$ & ${ }^{7} P^{o}$ & $3.93[-14]$ & $3 d^{5}{ }^{6} S^{e} 7 g$ & ${ }^{7} G^{e}$ & $2.62[-14]$ \\
\hline $3 d^{5}{ }^{6} S^{e} 8 f$ & ${ }^{7} F^{o}$ & $2.63[-13]$ & $3 d^{5}{ }^{6} S^{e} 8 f$ & ${ }^{7} F^{o}$ & $8.19[-14]$ & $3 d^{5}{ }^{6} S^{e} 8 g$ & ${ }^{7} G^{e}$ & $3.47[-14]$ & $3 d^{5}{ }^{6} S^{e} 5 g$ & ${ }^{7} G^{e}$ & $2.58[-14]$ \\
\hline $3 d^{5}{ }^{6} S^{e} 8 h$ & ${ }^{7} H^{o}$ & $2.42[-13]$ & $3 d^{5}{ }^{6} S^{e} 8 h$ & ${ }^{7} H^{o}$ & $7.27[-14]$ & $3 d^{5}{ }^{6} S^{e} 8 f$ & ${ }^{7} F^{o}$ & $3.44[-14]$ & $3 d^{5}{ }^{6} S^{e} 8 f$ & ${ }^{7} F^{o}$ & $2.27[-14]$ \\
\hline $3 d^{5}{ }^{6} S^{e} 7 h$ & ${ }^{7} H^{o}$ & $2.38[-13]$ & $3 d^{5}{ }^{6} S^{e} 7 h$ & ${ }^{7} H^{o}$ & $7.16[-14]$ & $3 d^{5}{ }^{6} S^{e} 9 g$ & ${ }^{7} G^{e}$ & $2.80[-14]$ & $3 d^{5}{ }^{6} S^{e} 8 g$ & ${ }^{7} G^{e}$ & $2.15[-14]$ \\
\hline $3 d^{5}{ }^{6} S^{e} 9 g$ & ${ }^{7} G^{e}$ & $2.32[-13]$ & $3 d^{5}{ }^{6} S^{e} 9 g$ & ${ }^{7} G^{e}$ & $7.13[-14]$ & $3 d^{5}{ }^{6} S^{e} 8 h$ & ${ }^{7} H^{o}$ & $2.66[-14]$ & $3 d^{5}{ }^{6} S^{e} 4 s$ & ${ }^{7} S^{e}$ & $1.78[-14]$ \\
\hline $3 d^{5}{ }^{6} S^{e} 9 h$ & ${ }^{7} H^{o}$ & $2.20[-13]$ & $3 d^{5}{ }^{6} S^{e} 9 h$ & ${ }^{7} H^{o}$ & $6.62[-14]$ & $3 d^{5}{ }^{6} S^{e} 7 h$ & ${ }^{7} H^{o}$ & $2.62[-14]$ & $3 d^{5}{ }^{6} S^{e} 9 g$ & ${ }^{7} G^{e}$ & $1.73[-14]$ \\
\hline $3 d^{5}{ }^{6} S^{e} 9 f$ & ${ }^{7} F^{o}$ & $1.95[-13]$ & $3 d^{5}{ }^{6} S^{e} 9 f$ & ${ }^{7} F^{o}$ & $6.08[-14]$ & $3 d^{5}{ }^{6} S^{e} 9 f$ & ${ }^{7} F^{o}$ & $2.56[-14]$ & $3 d^{5}{ }^{6} S^{e} 9 f$ & ${ }^{7} F^{o}$ & $1.69[-14]$ \\
\hline $3 d^{5}{ }^{6} S^{e} 10 h$ & ${ }^{7} H^{o}$ & $1.91[-13]$ & $3 d^{5}{ }^{6} S^{e} 10 h$ & ${ }^{7} H^{o}$ & $5.76[-14]$ & $3 d^{5}{ }^{6} S^{e} 9 h$ & ${ }^{7} H^{o}$ & $2.42[-14]$ & $3 d^{5}{ }^{6} S^{e} 6 p$ & ${ }^{7} P^{o}$ & $1.67[-14]$ \\
\hline $3 d^{5}{ }^{6} S^{e} 10 g$ & ${ }^{7} G^{e}$ & $1.88[-13]$ & $3 d^{5}{ }^{6} S^{e} 10 g$ & ${ }^{7} G^{e}$ & $5.76[-14]$ & $3 d^{5}{ }^{6} S^{e} 6 p$ & ${ }^{7} P^{o}$ & $2.40[-14]$ & $3 d^{5}{ }^{6} S^{e} 8 h$ & ${ }^{7} H^{o}$ & $1.55[-14]$ \\
\hline $3 d^{5}{ }^{6} S^{e} 6 p$ & ${ }^{7} P^{o}$ & $1.82[-13]$ & $3 d^{5}{ }^{6} S^{e} 6 p$ & ${ }^{7} P^{o}$ & $5.68[-14]$ & $3 d^{5}{ }^{6} S^{e} 4 s$ & ${ }^{7} S^{e}$ & $2.38[-14]$ & $3 d^{5}{ }^{6} S^{e} 7 h$ & ${ }^{7} H^{o}$ & $1.53[-14]$ \\
\hline Sum: & & $6.40[-12]$ & & & $1.98[-12]$ & & & $9.53[-13]$ & & & $1.05[-12]$ \\
\hline Total $=$ & & $1.08[-10]$ & & & $2.10[-11]$ & & & $6.86[-12]$ & & & $5.08[-12]$ \\
\hline$\%$ contribution & & $6 \%$ & & & $9 \%$ & & & $14 \%$ & & & $21 \%$ \\
\hline
\end{tabular}


estimated from the sum of the two rates. Their low- $T$ rates, valid over a small $T$ range, underestimate the recombination rate significantly, most likely due to the fact that they did not include any resonance contributions at low energies. Their DR rates (calculated using the Burgess general formula but possibly inaccurate oscillator strengths) are considerably higher, and overestimate the recombination rate by almost an order of magnitude near the high temperature peak. The asterisks in the figure are the DR rate coefficients by Hahn [7]. Fe III is one of the ions in the gap regions for which he did not have any available data for the empirical formula. Hence he deduced the formula from empirical predictions commenting that these values should be tested by new and improved calculations. His values agree with those of Woods et al.

Based on the accuracy of the close coupling approximations for the photoionization cross sections and DR collision strengths, the accuracy of the present $\alpha_{R}(T)$ values should be within $10 \%$ for most of the temperature range. At very low temperatures, $T \leqslant 100 \mathrm{~K}$, the uncertainty may be higher than $10 \%$ as the resolution of the near-threshold resonances in the photoionization cross sections is crucial. The uncertainty may also be higher in the very high temperature range where inner-shell ionization may be important but is not taken into account in the present work.

We also present the state-specific, partial recombination rate coefficients for some individual low- $n$ states at four temperatures: $\log _{10} T=2.0,3.0,3.7$, and 4.0, in Table II. These are the dominant contributors from the quintet and septet symmetries to the total $\alpha_{R}$. Their relative contributions to $\alpha_{R}$, however, change with temperature because of the varying contribution from autoionizing resonances in $\sigma_{\mathrm{PI}}$ with photon energy. As explained in Ref. [18], the rates in Table II should be equal to the total state-specific recombination rate coefficient to the individual states at the temperatures considered, since the high- $n$ DR contribution does not dominate until about $\log _{10} T \approx 4.7$. Table II shows that the quintets contribute more to $\alpha_{R}$ than the septets.

\section{CONCLUSION}

The total recombination rate coefficients for the recombined ion Fe III are obtained in the close coupling approximation employing a unified treatment. To our knowledge this is the first detailed calculation for the recombination rate coefficients of Fe III. Present results differ considerably from the currently used values obtained by Woods et al. [4]. Their $\mathrm{RR}$ rates at low temperatures underestimate the recombination rate, but the huge difference appears at higher temperatures where their DR rates are up to an order of magnitude higher than the present rates close to the high temperature peak. Present calculations are also an application of the unified treatment to a complex atomic system from the iron group, involving large eigenfunction expansions for the recombining ion to account for the strong electron correlation effects and coupling. Similar calculations are now in progress for Fe I and Fe II. To our knowledge, there exists no other detailed calculations for Fe III recombination rates.

\section{ACKNOWLEDGMENTS}

I would like to thank Professor Anil K. Pradhan for contributions (supported by NSF Grant No. PHY-9421898). This work was supported by NASA Grants No. NAGW-3315 and No. NAS-32643. The computational work was carried out on the Cray YMP at the Ohio Supercomputer Center.
[1] S.N. Nahar and A.K. Pradhan, Phys. Rev. Lett. 68, 1488 (1992).

[2] S.N. Nahar and A.K. Pradhan, Phys. Rev. A 49, 1816 (1994).

[3] S.N. Nahar and A.K. Pradhan, Astrophys. J. 447, 966 (1995).

[4] D.T. Woods, J.M. Shull, and C.L. Sarazin, Astrophys. J. 249, 399 (1981).

[5] A. Burgess, Astrophys. J. 141, 1588 (1965).

[6] D. Abbott, Ph.D. thesis, University of Colorado, 1978.

[7] Y. Hahn, J. Quant. Spectrosc. Radiat. Transfer 41, 315 (1989).

[8] M. Arnaud and J.R. Raymond, Astrophys. J. 398, 394 (1992).

[9] R.H. Bell and M.J. Seaton, J. Phys. B 18, 1589 (1985).

[10] S.N. Nahar, Phys. Rev. A 53, 1545 (1996).

[11] M. J. Seaton, J. Phys. B 20, 6363 (1987); K.A. Berrington, P.G. Burke, K. Butler, M.J. Seaton, P.J. Storey, K.T. Taylor, and Yu. Yan, J. Phys. B 20, 6379 (1987).
[12] D.G. Hummer, K.A. Berrington, W. Eissner, A.K. Pradhan, H.E. Saraph, and J.A. Tully, Astron. Astrophys. 279, 298 (1993).

[13] R.F. Reilman and S.T. Manson, Astrophys. J. Suppl. 40, 815 (1979).

[14] D.E. Osterbrock, Astrophysics of Gaseous Nebulae and Active Galactic Nuclei (University Science Books, Mill Valley, CA, 1989).

[15] P.M.J. Sawey and K.A. Berrington, J. Phys. B 25, 1451 (1992).

[16] P.J. Storey and D.G. Hummer, Comput. Phys. Commun. 66, 129 (1992).

[17] D.G. Hummer, Mon. Not. R. Astron. Soc. 268, 109 (1994).

[18] S.N. Nahar, Astrophys. J. Suppl. 101, 423 (1995).

[19] The state-selective recombination rate coefficients of Fe III may be obtained from the author (electronic address: nahar@seaton.mps.ohio-state.edu). 